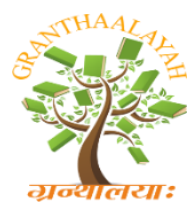

\author{
INTERNATIONAL JOURNAL OF RE
GRANTHAALAYAH \\ A knowledge Repository
}

Science

\title{
HEALTH INFORMATION MANAGEMENT (HIM) IN THE CONTROL OF MEDICAL CLAIMS ERRORS
}

\author{
Kashef K. Alshaban ${ }^{1}$, Lina Almasri ${ }^{2}$ \\ ${ }^{1}$ Consultant Oral \& Maxillo-Facial Surgeon, Faculty of Medical \& Health Sciences, Emirates \\ College of Technology/Abu Dhabi - UAE, \\ ${ }^{2}$ Healthpoint Hospital/Abu Dhabi - UAE
}

\begin{abstract}
Preparing and submitting medical claims to health care providers is a crucial component in hospital finance. Owing to the complexity of the process, medical claims errors may result in financial issues.

Our objective is to examine the process of preparing and submitting medical claims in the distinguished Healthpoint Hospital/Abu Dhabi, also the reasons for rejection of medical claims so as to focus in control hospital's medical claims errors.

The cases of claim rejection between the years 2014-2018 according to the hospital records are as follows: 2018 (January - May) 5\%, 2017 4.4\%, 2016 8\%, 2015 \& 2014 24\%. Other cases of medical claims regarding Vitiligo (37 cases), obesity (14 cases), and Audiometry (23 cases) are also reviewed.

It seems that the most common medical billing errors are related to technical and nontechnical errors.

The strategy of controlling medical claims errors is designed in the hospital by applying key performance indicators (KPI) that demonstrate how effectively the hospital is achieving the key business objectives. The result proves that the strongest electronic system and applying the new business strategies led to reducing the rejections of medical claims due to mistakes.
\end{abstract}

Keywords: Medical Claims; Rejection of Medical Claims; Reimbursement; Key Performance Indicator (KPI).

Cite This Article: Kashef K. Alshaban, and Lina Almasri. (2020). "HEALTH INFORMATION MANAGEMENT (HIM) IN THE CONTROL OF MEDICAL CLAIMS ERRORS." International Journal of Research - Granthaalayah, 8(1), 327-335.

\section{Introduction}

The health information management (HIM) specialty turns the concept of health management from the tradition form "paper forms" to electronic forms. It is "the practice of acquiring, analyzing, and protecting digital and traditional medical information vital to providing quality patient care."(AHIMA) ${ }^{1}$ HIM helps in analyzing the health record for billing and reimbursement purposes 
by applying electronic health records (EHR), which is simply an electronic system of a patient's medical history which facilitates access to patient's medical records by health providers, researchers, and the rest of hospital departments. (Shapland) ${ }^{2}$

The medical management software introduced by the health insurance department in the healthcare facilities leads to efficiently manage a large number of claims and submit them successfully. The process of submitting medical claims involves a request from healthcare providers that contracting with payers (health insurance companies) for reimbursement of the medical service obtained to the patients. (AAPC) ${ }^{3}$ The whole process of managing claims, payment and billing referred to as revenue cycle management. (Belliveau, 2016)

The process is started when the patients visit the health care provider and create or update the medical record. (Medical Billing and Coding online $)^{5}$ The subsequent process after the doctor sees the patients and assigns the diagnosis and procedures codes is the medical coders organize a report summary using ICD-10 (international classification for disease version 10) codes and CPT (current procedural technology) codes which are helpful in determining health insurance companies coverage limits. Finalize the medical submission team will review the claims and transmit it to the payer (health insurance companies) electronically. (Medical Billing and Coding online) ${ }^{5}$

The probability of having mistaken during the process of preparing the medical claims exists although the electronic system. A study by Nerdwallet about Medicare claims in 2013 found that the average medical claims errors are $49 \%$ (Lamontagne, 2014) ${ }^{6}$ and that some medical centers messed up on more than $80 \%$ of claims to Medicare. (HOBSON, 2015) ${ }^{7}$ In addition, the American Medical Association found in a study that one in 10 bills paid by private health insurance has mistaken (Lipka, 2013) ${ }^{8}$. In fact, some errors in medical claims can directly be treated simply by reviewing information of the patients and resubmit it, but on the other side, there is an error that causes delay payments, fines and lost revenue. (AAPC, 2016) ${ }^{9}$

\section{Materials and Methods}

To understand and define the reasons for rejection of medical claims and the strategies used to control the rejection rates in (Healthpoint Hospital/ Abu Dhabi/ UAE) Quantitative and Qualitative methods were used. Initial investigations through the quantitative method give a deeper and broad understanding of the process, by doing a focus group and discussions with the IT, coding \& insurance departments, while the qualitative method is used to collect data to evaluate the reasons for medical claims rejection. The total percentages of final medical claims rejection were reviewed from 2014 until 2018 with defined reasons; besides it reviewed the total rejections of some diagnosis and procedures includes 37 of vitiligo claims, 14 of obesity claims and 23 of services of audiometry test claims. Key performance indicator (KPI) that is applied in the hospital is essential in the strategic planning to monitor, evaluate, and control the rejection rates of medical claims so as to keep it within acceptable ranges.

Our main objective was to collect data from saving claims in computers and systems regarding the reasons for rejection claims with statistics across different services. According to analyze step, it used tables to present the results. For a deep analysis of the root of rejections, it will be identified 
and displayed in columns and pie charts that visualize the most critical reasons for rejection with percentages clearly.

Results In our study, data were gathered from the Health Information Technology Department (HIT) and Coding \& Insurance Department of Healthpoint Hospital. The dataset consisted of related records generally from 2014 to 2018 and in detail samples from January to May 2018. In 2018, the total transactions until now are 115000, nearly 5750 transactions are rejected.

The main 3critical rejection reasons were determined:

1) Medical Necessity: it means that accepted health care services and supplies provided by health care providers based on clinical evidence of standards of care. So, some patients have a lack of justifications that lead to rejecting the claim.

2) Agreement Contract: it is the agreement between hospitals and insurance companies about the policies of payments for each insurance plan like covered, partially covered and not covered procedures.

3) Surgery Approval-In-Patient: especially for plastic surgery due to not medical situations and does not affect the patient's lives.

The rejected claims in Healthpoint Hospital from 2014 to May 2018 are shown in table 1.

\begin{tabular}{|l|l|}
\hline Year & Table 1: (Total rejections \%) \\
\hline 2014 & 24 \\
\hline 2015 & 24 \\
\hline 2016 & 8 \\
\hline 2017 & 4.4 \\
\hline 2018 (Jan-May) & 5 \\
\hline
\end{tabular}

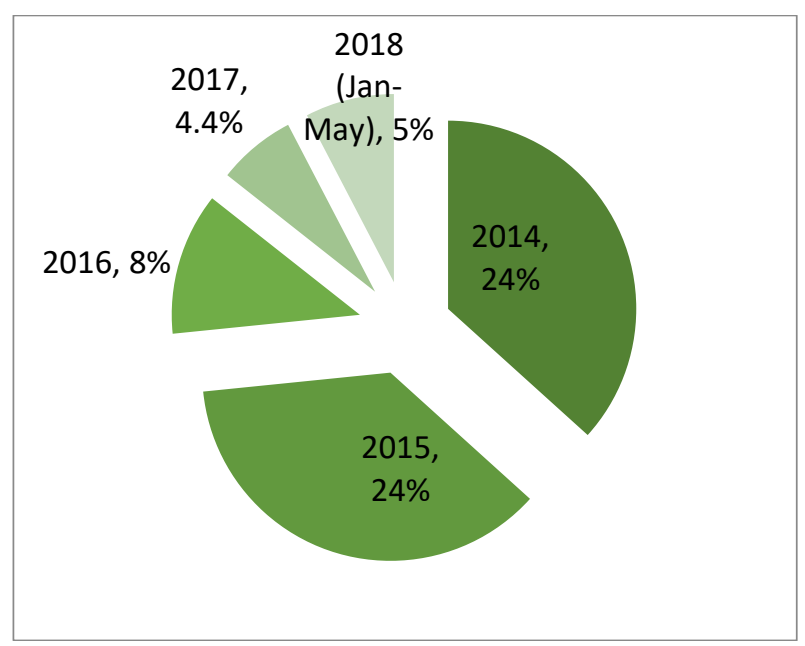

Figure 1: (Rejection Rates \%)

The Rest of errors like missing information, mismatch diagnosis with procedures codes, insurance not cover services and expired insurance cards have occurred but not much, which do not affect the final rejection rates. Examples: 


\section{Vitiligo Medical Claims}

Table 2: (Vitiligo medical claims statistics)

\begin{tabular}{|l|l|l|}
\hline No. of claims & Rate (\%) & Status \\
\hline 34 & 92 & paid \\
\hline 3 & 8 & rejected \\
\hline 37 & 100 & \multicolumn{1}{|c}{} \\
\cline { 1 - 2 } & \multicolumn{2}{|l|}{} \\
\cline { 1 - 2 } & &
\end{tabular}

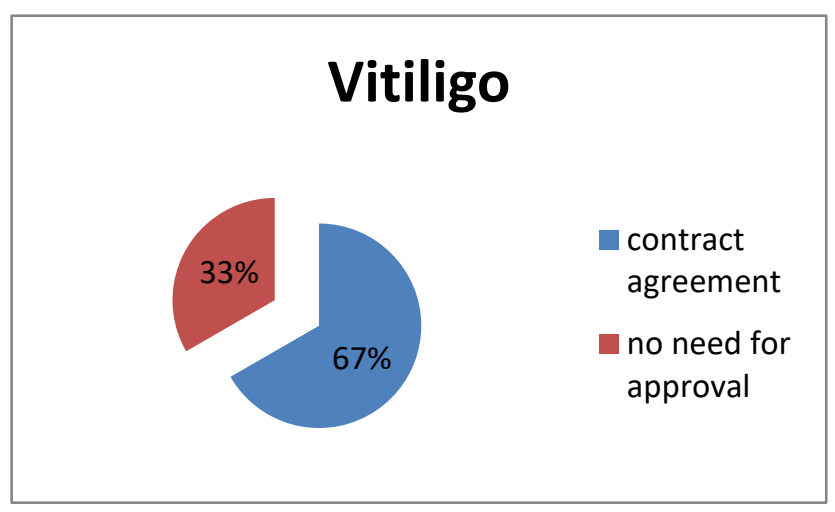

Figure 2: (Vitiligo rejection statistics)

The 3 claims that were rejected due to: (Figure 2)

- 2 rejected claims are due to the contract agreement that diagnosis and services are not covered by the insurance.

- 1 rejected claim is due to the claim that do not need authorizations/approval.

\section{Obesity Medical Claims}

Table 3: (Obesity medical claims statistics)

\begin{tabular}{|c|l|l|}
\hline No. Of Claims & Rate $(\boldsymbol{\%})$ & Status \\
\hline 4 & 28.6 & paid \\
\hline 10 & 71.4 & rejected \\
\hline 14 & 100 & \multicolumn{1}{|c}{} \\
\cline { 1 - 2 } & \multicolumn{2}{|l|}{}
\end{tabular}

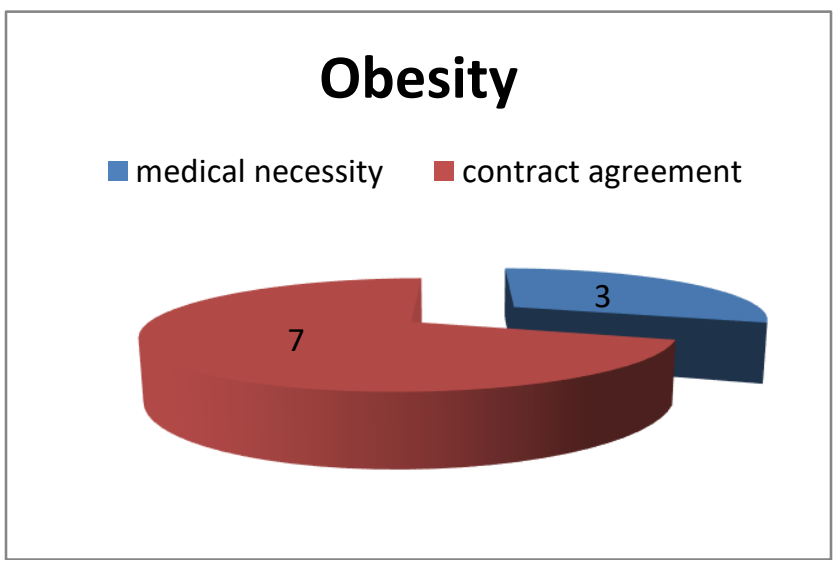

Figure 3: (Obesity rejection statistics) 
The 10 claims that were rejected due to: (Figure 3)

- 3 rejected claims are due to no indications of medical necessity.

- 7 rejected claims are due to the contract agreement between the hospital and insurance company.

\section{Audiometry Medical Claims}

Table 1: (Audiometry medical claims statistics)

\begin{tabular}{|l|l|l|}
\hline No. Of Claims & Rate (\%) & Status \\
\hline 0 & 0 & paid \\
\hline 23 & 100 & rejected \\
\hline 23 & 100 & \multicolumn{1}{|c}{} \\
\cline { 1 - 2 } & &
\end{tabular}

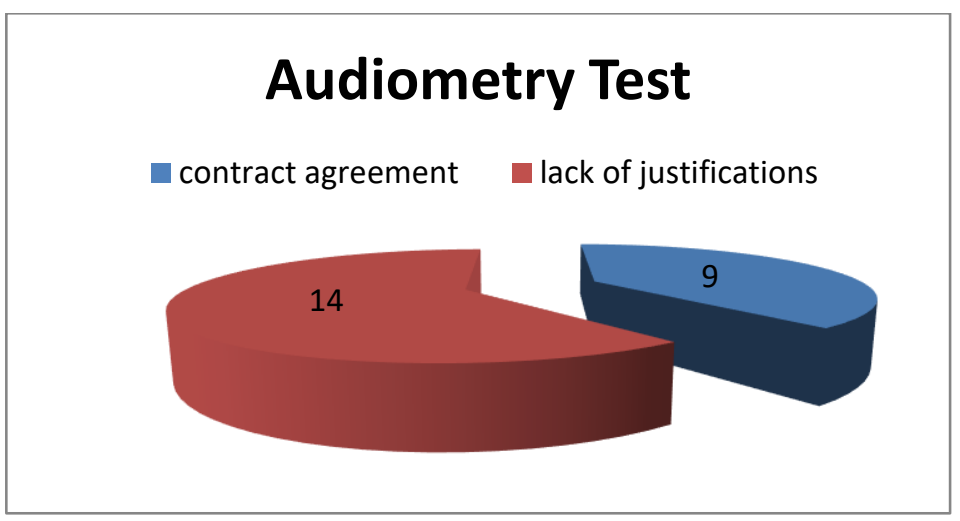

Figure 4: (Audiometry test rejection statistics)

The 23 claims that were rejected due to: (Figure 4)

- 9 rejected claims are due to the contract agreement between the hospital and insurance company.

- 14 rejected claims are due to a lack of justifications of hearing loss.

\section{Controlling Medical Claims Rejections}

Key performance indicator (KPI) is the main strategy applied in the hospital by the coding and insurance department to control the rejection rates of medical claims and the process of submitting. KPI as an evaluation of the success of the hospital or for certain actions like the process of coding and insurance. Here, a summary of performance indicators (Table 5).

Table 2: (KPI measurement)

\begin{tabular}{|l|l|l|}
\hline $\begin{array}{l}\text { Core Performance } \\
\text { Metric }\end{array}$ & Measured Metric & Target \\
\hline \multirow{4}{*}{ Financial } & Initial rejection rate & $\begin{array}{l}11 \% \text { rejection rate over gross } \\
\text { revenue }\end{array}$ \\
\cline { 2 - 3 } & Final rejection rate & $\begin{array}{l}5-6 \% \text { rejection rate over gross } \\
\text { revenue }\end{array}$ \\
\cline { 2 - 3 } & Coding optimization & 1.2 Million per month \\
\hline & Timely approvals/authorization & 2 weekdays \\
\hline
\end{tabular}




\begin{tabular}{|l|l|l|}
\hline \multirow{4}{*}{ Operational efficiency } & Timely claim submission & 24 hours from billing month \\
\cline { 2 - 3 } & Timely re-submission & 24 hours from received date \\
\cline { 2 - 3 } & Coding accuracy & $86 \%$ audit score \\
\cline { 2 - 3 } Quality & $\begin{array}{l}\text { Rejection analysis \& } \\
\text { communications }\end{array}$ & 24 hours from the billed date \\
\cline { 2 - 3 } & Department training & Each quarter \\
\hline Initiatives/projects & New payers inclusions & 3-5 Networks \\
\hline
\end{tabular}

\section{Discussion}

The flow of information (from the time a patient is admitted to the hospital to his/her discharges are submitted) is a critical factor in providing efficient patient care and billing for services rendered during the patient visit. It requires various departments to perform functions cautiously.

Patient care services are coded and charges are entered by specified personnel in various clinical departments and by HIM. The content of the presented data is critical to successfully submit medical claims. The process of documenting, billing, and preparing medical claims that applied in Healthpoint Hospital is taken from the USA, which is summarized in:

- Create a file for the patient with preliminary demographic information;

- Each patient should complete some consent forms, and the eligibility of insurance plan is confirmed;

- A medical report is prepared (on discharge) that includes diagnosis, procedures, and treatment for each patient.

- Bills are prepared by the cashier and send to the medical coders. (medical billing and coding $)^{10}$

- To improve reimbursements, we should focus on the reasons for medical claims rejection which include: recurrent errors, insurance companies contract agreement, and patient health situation that described in the claim is not enough to accept the claims.

\section{Recurrent Errors}

- Missing information and duplicate submit of claim,

- Errors in both upcoding and down coding following the errors of lacking documentation (AMA, 2013) $)^{11}$

\section{Insurance Companies}

- In some cases, some insurance companies when they renew the insurance plan, change the member ID number. In these cases, the claims are rejected due to an expired insurance plan.

- Changing of insurance status due to canceling of plan or changes of coverage (NCG Medical, Health Department Perspective) ${ }^{12}$ 


\section{Patient Health Situation}

1) Contract agreement rejections: such as Vitiligo medical claims (in our study, 2 rejections from 3 transactions). The health insurance companies for some plans do not cover the diagnosis (consultation) or services for treatments by virtue of long journeys and high cost of treatment.

2) Medical necessity: in Healthpoint Hospital, two examples that have supported this reason which is: 3 rejected claims from 10 rejected transactions of obesity as mentioned in figure 3 , and 14 rejected claims from 23 rejected transactions of audiometry test as seen in figure 4. This type of rejection may include sub causes for instance lack of justifying in the medical record, the age of patient and health status. The explaining is provided by real examples:

- For the audiometry test, the rejection reason was whether medically justified for hearing loss to check the activity of the cochlear amplifier or medically justified for hearing loss to check conductive or sensorineural loss.

- For obesity, the rejection reason was whether the age of the patient or no medical indication:

3) One of the most common rejections in Healthpoint Hospital is inpatients approvals, especially for plastic surgery. However, plastic surgery is provided primarily for people victims of accidents like a car accident and burn.

4) Many people exploit this type of surgery to change their body appearances like otoplasty, liposuction, dermabrasion, and Mastopexy without the need to do. (CBS News) ${ }^{13}$

5) In 2018, according to KPI, the target of medical claims error should be between 5-6\% yearly. The results found according to the hospital records are as follows from 2014 to 2017 and are reduced from $24 \%$ to 4.4 in Healthpoint Hospital.

6) During Jan to May 2018 Healthpoint Hospital had 115000 transactions of medical claims, had nearly 5750 different rejections error types, while according to a research that did in Hacettepe University in Turkey on January to March 2012 they checked 48000 transactions, had nearly 2500 different error types which are the most recurrent error in judicial reports which occur in emergency department, that it is must collect legal reports to prove situations of patients (Erdi- et al., 2013) ${ }^{\mathbf{1 4}}$

\section{Conclusion and Recommendations}

Controlling medical claims errors can be eliminated. It will, however, take time, money and combined efforts on the part of many employees in the hospital. This research paper covered the process of preparing medical claims to control medical claims mistakes and offered recommendations on how providers can work towards lowering their error rates. Whether technical or nontechnical errors incur delayed payment, denials, client complaints or even just lost productivity, medical claims errors hurt the health care providers. Eliminating errors is critical to the bottom line, but begins with understanding the most common errors leading to rejected claims. The stated overall and specific goals for this study and the design of it to achieve those goals largely reflect the controlling missions of medical claims errors according to years in Healthpoint Hospital. In the broadest terms, the Healthpoint Hospital goals and design are responsive to all regulations that help to control medical claims errors. 
According to the areas that examined in Healthpoint Hospital, as represented in the research paper, it is not, or may not be, optimal for achieving the maximum performance of preparing and submitting medical claims without errors. Here, a recommendation is stated to reach to optimality:

- Improving the HIM department will improve the documentation of medical records.

- The efficiency of insurance and coding staff.

- Applying a second strongest electronic system for patient care service "Cerner" that checks automatically every day the patients' ID.

- Applying the strongest electronic system for submitting medical claims "Globis" which has advanced features.

- Set a qualified staff for internal audit.

- Applying KPI accurately.

\section{Data Availability}

lina_almasri@outlook.com

\section{Conflicts of Interest}

"The authors declare that there is no conflict of interest regarding the publication of this paper."

\section{Funding Statement}

None

\section{Acknowledgments}

We would like to thank the staff of Healthpoint Hospital/Abu Dhabi for their cooperation and help in providing us valuable information according to their respective fields.

\section{References}

[1] AHIMA. (n.d.). Retrieved from Health Information Management: http://www.ahima.org/careers/healthinfo.

[2] Shapland, M. (n.d.). Study.com. Retrieved from Health Information Management: Definition, Use \& Ethics: https://study.com/academy/lesson/health-information-management-definition-useethics.html.

[3] AAPC. (n.d.). Retrieved from Medical Billing: https://www.aapc.com/medical-billing/medicalbilling.aspx.

[4] Belliveau, J. (2016, August 2). Rev cycle intelligence. Retrieved from 4 Medical Billing Issues Affecting Healthcare Revenue Cycle: https://revcycleintelligence.com/news/4-medical-billingissues-affecting-healthcare-revenue-cycle.

[5] Medical Billing and Coding Online. (n.d.). Retrieved from The Medical Billing Process: http://www.medicalbillingandcodingonline.com/medical-coding-for-billers/

[6] Lamontagne, C. (2014). NerdWallet Health Study: Medical Debt Crisis Worsening Despite Policy Advances. USA: NerdWallet Health, Oct.8.2014

[7] HOBSON, K. (2015, April 21). Retrieved from The Danger Lurking in Your Medical Bills-Money: http://time.com/money/3819569/medical-billing-errors/

[8] Lipka, M. (2013). How to handle medical billing errors. Reuters, Wealth July 3, 2012

[9] AAPC. (2016, April 1). Retrieved from Conquer Common Billing Errors: https://www.aapc.com/blog/34359-conquer-common-billing-errors/. 
[10] Medical billing and coding. (n.d.). Retrieved from Insurance and the insurance claims process: https://www.medicalbillingandcoding.org/insurance-claims-process/.

[11] AMA. By Thomas Sullivan (2013). American Medical Association National Health Insurer Report Card. USA: American Medical Association, Jul 18, 2013

[12] NCG Medical, Inc. 2016 (A Local Health Department Perspective). Common Medical Billing Mistakes and Solutions. USA: Illinois Public Health Association.

[13] CBS News. (n.d.). Retrieved from 10 most popular plastic surgery procedures: https://www.cbsnews.com/pictures/10-most-popular-plastic-surgery-procedures/5/

[14] Erdi, D. et al. (2013). Improving Hospital Billing Processes for Reducing Costs of Billing Errors. Ankara, Turkey: Hacettepe University. (ENBIS) 13 the Conference, 15-19 September 2013.

\footnotetext{
*Corresponding author.

E-mail address: kashef.alshaban@ect.ac.ae/lina_almasri@outlook.com
} 